\title{
Propofol ensures a more stable A-line ARX index than thiopental during intubation
}

\author{
[Le propofol assure un index «A-line ARX» plus stable que le thiopental pendant
}

l'intubation]

Jee-Ching Hsu MD PhD, ${ }^{*}$ Ching-Yue Yang MD, ${ }^{*}$ Lai-Chu See PhD, $†$ Jiin-Tarng Liou MD, ${ }^{*}$ Fu-Chao Liu MD, * Jong-Jang Hwang MD, ${ }^{*}$ Wun-Chin Wu PhD $\ddagger$ Ping-Wing Lui MD PhD*

Backgound: The A-line autoregressive modelling with exogenous input index (AAI) is a new method of assessing depth of anesthesia. We examined the effects of tracheal intubation on the AAl and hemodynamics during induction of anesthesia with propofol compared with thiopental in patients aged over $50 \mathrm{yr}$.

Methods: 40 patients scheduled for a laminectomy, posterior spinal fusion, vertebroplasty, or total hip replacement, ASA physical status I or II and aged over $50 \mathrm{yr}$, were randomly divided into two groups. Thiopental $5 \mathrm{mg} \cdot \mathrm{kg}^{-1} \mathrm{iv}$, fentanyl $2.5 \mu \mathrm{g} \cdot \mathrm{kg}^{-1} \mathrm{iv}$, and rocuronium $0.7 \mathrm{mg} \cdot \mathrm{kg}^{-1}$ iv were used in the thiopental group $(n=20)$ for anesthetic induction; the same protocol was used in the propofol group $(n=20)$ except that $2 \mathrm{mg} \cdot \mathrm{kg}^{-1}$ propofol iv was given instead of thiopental. The AAI, non-invasive blood pressure, and heart rate were measured every minute before induction for three minutes, at 1.5 min post-induction, and then each minute post-intubation for eight minutes.

Results: The AAI increased significantly at one and two minutes after intubation in the thiopental group (to $56.5 \pm 18.6$ at I $\mathrm{min}$ and $44.7 \pm 18.7$ at $2 \mathrm{~min}$ after intubation vs $19.9 \pm 7.5$ at $1.5 \mathrm{~min}$ after induction; $P<0.05)$. Thereafter, AAl values gradually decreased three minutes after intubation. The AAl was inhibited continuously after intubation in the propofol group, and no significant elevation was seen.

Conclusion: Our results, using the AAl to monitor anesthetic depth during induction and tracheal intubation, suggest that at equipotent doses propofol provided a more stable level of anesthesia than did thiopental.
Objectif : La modélisation autorégressive A-line avec index des potentiels évoqués (AAl) est une nouvelle méthode d'évaluation de la profondeur d'anesthésie. Nous avons vérifié les effets de l'intubation endotrachéale sur l'AAl et l'hémodynamique pendant l'induction de l'anesthésie au propofol ou au thiopental chez des patients de plus de 50 ans.

Méthode : Quarante patients, d'état physique ASA I ou II et de plus de 50 ans, devant subir une laminectomie, une arthrodèse vertébrale postérieure, une vertébroplastie ou une arthroplastie totale de la hanche, ont été divisés au hasard en deux groupes. On a utilisé : 5 $\mathrm{mg} \cdot \mathrm{kg}^{-1}$ iv de thiopental, $2,5 \mu \mathrm{g} \cdot \mathrm{kg}^{-1}$ de fentanyl iv et $0,7 \mathrm{mg} \cdot \mathrm{kg}^{-1}$ iv de rocuronium dans le groupe thiopental $(n=20)$ pour l'induction de l'anesthésie ; le même protocole a été utilisé dans le groupe propofol $(n=20)$ sauf que $2 \mathrm{mg} \cdot \mathrm{kg}^{-1}$ de propofol iv ont remplacé le thiopental. L'AAl, la tension artérielle par mesure non effractive et la fréquence cardiaque ont été notés toutes les minutes avant l'induction pendant trois minutes, à I, 5 min après l'induction et à chaque minute après l'intubation pour huit minutes.

Résultats : L'AAl s'est élevé significativement à une et deux minutes après l'intubation avec le thiopental (56,5 $\pm 18,6$ à I min et 44,7 \pm 18,7 à 2 min après l'intubation vs 19,9 \pm 7,5 à 1,5 min après l'induction, $P<0,05)$. Puis, les valeurs de l'AAl ont graduellement diminué trois minutes après l'intubation. L'AAl a été inhibé de façon continue après l'intubation dans le groupe propofol et aucune élévation significative n'a été signalée.

Conclusion : Avec l'usage de l'AAl pour évaluer la profondeur de l'anesthésie pendant l'induction et l'intubation endotrachéale, nous avons constaté qu'à doses équivalentes, le propofol fournit un niveau d'anesthésie plus stable que le thiopental.

From the Department of Anesthesiology, Chang Gung Memorial Hospital;* the Department of Public Health, Chang Gung University, $\dagger$ Taoyuan; and the Department of Electronic Engineering, National Penghu Institute of Technology; Penghu, Taiwan, ROC.

Address correspondence to: Dr. Ching-Yue Yang, Department of Anesthesiology, Chang Gung Memorial Hospital, No. 5 Fu-Shing

Street, Kweishan, Taoyuan, Taiwan 333, R.O.C. Phone: 886-3-3281200, ext. 3621; Fax: 886-3-3281200 ext, 2793;

E-mail 1: yangcy@adm.cgmh.org.tw; E-mail 2: yangcy88@seed.net.tw

Assessed July 30, 2004.

Accepted for publication March 3, 2005.

Final revision accepted April 6, 2005. 
M

IDDLE-LATENCY auditory evoked potentials (AEP) have been reported to correlate well with depth of anesthesia in patients undergoing general anesthesia. ${ }^{1-3}$ However, the waveforms of AEP are difficult to extract and interpret in clinical situations. This monitoring method has not been introduced as a standard for evaluation of anesthetic depth., ${ }^{4,5}$ Recently, Jensen $e t$ al. developed a new method for rapid extraction of AEP by using advanced signal processing (autoregressive modelling with exogenous input, ARX). ${ }^{6}$ This process facilitates a rapid extraction of AEP, within 15 repetitions of click stimulus, resulting in only a six-second response delay time. A new variable, called the A-line ARX index (AAI), is then calculated from this rapidly extracted AEP wave. This new technology was incorporated in a recently commercialized system called the A-line ${ }^{\mathrm{TM}}$ AEP monitor (Danmeter, Odense, Denmark). Recent articles have reported that the AAI is an accurate indicator of the level of anesthesia and allows its on-line assessment. ${ }^{7,8}$

Our previous study reported that endotracheal intubation after a thiopental induction in elderly patients may induce temporary hypertension and tachycardia even in the amnesic phase. ${ }^{9}$ Because the extent of the blood pressure increase is so high, it may increase morbidity due to cardiac and neurological adverse effects. If we use propofol for intubation in elderly patients, there is significantly less post-intubation hypertension and tachycardia. How does anesthetic depth change when using these agents for anesthetic induction and intubation, and how does the anesthetic depth correlate with post-intubation hypertension and tachycardia? The answers are not clear at present. We hypothesized that propofol induces deeper anesthesia than does thiopental, so propofol should produce less hypertension and tachycardia. The objective of this study was to compare the anesthetic depth during induction of anesthesia with either propofol or thiopental for patients aged over 50 yr. The anesthetic depth was assessed with the AAI.

\section{Methods}

The experiment was approved by the Committee of Human Studies at Chang Gung Memorial Hospital. Verbal consent for participation in the study was obtained from all patients prior to randomization. The non-invasive measurements and risks and benefits of A-line ${ }^{\mathrm{TM}}$ AEP monitoring for anesthesia were fully explained as part of the consent procedure. A sample size of 40 (20 received thiopental and 20 received propofol) was calculated for this study based upon our previous finding of the difference in heart rate $(\mathrm{HR})=$
13 beats $\cdot \min ^{-1}\left(\right.$ standard deviation $=15$ beats $\left.\cdot \mathrm{min}^{-1}\right)$ at one minute after tracheal intubation between two groups, ${ }^{9}$ significant level $=0.05$, statistical power $=$ 0.8 . Forty patients scheduled for a laminectomy $(n=$ 19), posterior spinal fusion $(n=11)$, vertebroplasty $(n$ $=4)$, or total hip replacement $(n=6)$, with a physical status of ASA I or II and aged over $50 \mathrm{yr}$, were included in the study. Patients who had central nervous or cardiovascular disorders were excluded. A doubleblind, randomized study protocol was used. Patients were randomly assigned to either the thiopental or propofol group according to which lot they drew from an opaque box containing 40 lots, 20 for thiopental and 20 for propofol. Measurements were recorded by a physician or technician who was unaware of study group assignment. No premedication was given. The A-line ${ }^{\mathrm{TM}}$ AEP monitor (Danmeter, Odense, Denmark) a non-invasive blood pressure (NIBP) system and electrocardiograph were installed before the experiment. The AAI, mean blood pressure (MBP), and HR were recorded every minute for three minutes as control values. Anesthesia was induced one minute after the last recording. Thiopental $5 \mathrm{mg} \cdot \mathrm{kg}^{-1} i v$, fentanyl $2.5 \mu \mathrm{g} \cdot \mathrm{kg}^{-1} i v$, and rocuronium $0.7 \mathrm{mg} \cdot \mathrm{kg}^{-1}$ iv were administered with the patient breathing $\mathrm{O}_{2}$ (4 $\left.\mathrm{L} \cdot \mathrm{min}^{-1}\right)$ through a face mask in the thiopental group $(n=20)$; the same protocol was used in the propofol group $(n=20)$, except that $2 \mathrm{mg} \cdot \mathrm{kg}^{-1}$ propofol were given instead of thiopental. One and a half minutes after the drugs had been injected, the AAI, NIBP, and HR were recorded as post-induction values. Endotracheal intubation was performed immediately thereafter. Patients who experienced a difficult intubation or for whom intubation was attempted more than two times were excluded. Immediately after intubation, anesthesia was maintained with $2 \mathrm{~L} \cdot \mathrm{min}^{-1} \mathrm{~N}_{2} \mathrm{O}$, $2 \mathrm{~L} \cdot \mathrm{min}^{-1} \mathrm{O}_{2}$, and sevoflurane. Sevoflurane was administered at a $5 \%$ concentration when systolic blood pressure was $>150 \mathrm{mmHg}$, at a $2.5 \%$ concentration when systolic blood pressure was between 100 and $150 \mathrm{mmHg}$, and at a $1.25 \%$ concentration when systolic blood pressure was $<100 \mathrm{mmHg}$. In the meantime, the AAI, NIBP, and HR were measured every minute for eight minutes after intubation. Thereafter, the remainder of anesthetic care was at the discretion of the attending anesthesiologist.

\section{Data analysis}

Data are presented as the mean and standard deviation (SD). Two-way analysis of variance (ANOVA) with repeated measures was used to examine how continuous variables (such as AAI, MBP, and HR) differed within the study period between the two study 
groups. Interactions between time and study groups were examined first. Tukey's HSD multiple comparisons were made to locate where differences existed. The mean square error was used for multiple comparisons. Computations were carried out in the ANOVA model with the main effect and interactions according to Hines's report. ${ }^{10}$ All $P$ values presented were twosided, and the significance level was 0.05 .

\section{Results}

Two-way ANOVA with repeated measures reveals that interaction between time and the study groups was significant for AAI $(P<0.001)$ and MBP $(P<0.001)$ but not for HR $(P=0.255)$. Hence, levels of AAI, MBP, HR at various times between the two study groups were compared using Tukey's HSD multiple comparison.

\section{Response to anesthetic induction}

Before anesthetic induction, MBP, AAI, and HR were similar in the thiopental and propofol groups (Figures 1 and 2). The AAI decreased in all groups $1.5 \mathrm{~min}$ after thiopental or propofol induction $(83.2 \pm 8.7 \mathrm{vs}$ $19.9 \pm 7.5$ in the thiopental group and $82.4 \pm 11$ vs $10.8 \pm 4.6$ in the propofol group, $P<0.05$ respectively, Figure 1). As for MBP and HR, only MBP in the propofol group was significantly reduced after iv induction $(P<0.05$, Figure 2$)$. There was no significant change in MBP in the thiopental group or HR in either group after anesthetic induction.

\section{Response to intubation}

After intubation, AAI values increased significantly at one and two minutes after intubation in the thiopental group when compared with AAI values in the propofol group $(56.5 \pm 18.6$ at $1 \mathrm{~min}$ and $44.7 \pm 18.7$ at $2 \mathrm{~min}$ after intubation in the thiopental group vs $13.9 \pm 4.2$ at $1 \mathrm{~min}$ and $12.8 \pm 4.7$ at 2 min after intubation in the propofol group, $P<0.05$, Figure 1 ). Thereafter, AAI values gradually decreased in the thiopental group (Figure 1). However, AAI values remained low after intubation in the propofol group (Figure 1). Intubation was not associated with an increase in MBP in the propofol group $(P>0.05$, Figure 2). In the thiopental group, intubation produced a significant elevation in blood pressure one minute after intubation when compared with control $(P<0.05$, Figure 2). MBP in the thiopental group was also higher than that in the propofol group from one to four minutes after intubation $(P<0.05$, Figure $2)$. Intubation was not associated with any change in HR in the propofol group when compared with control $(P>0.05$, Figure 2$)$. However, HR was signifi-

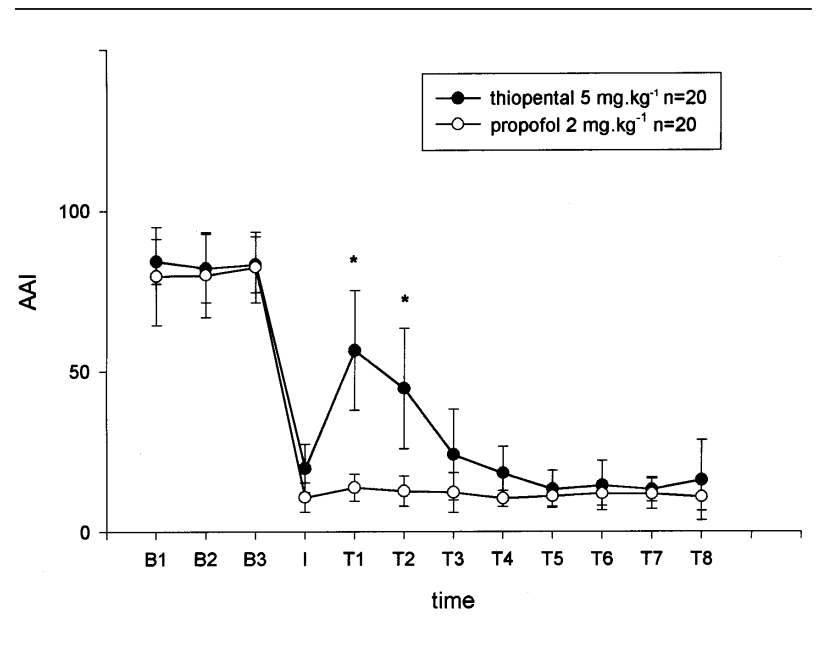

FIGURE 1 Time course of the A-line autoregressive modelling with exogenous input index (AAI) before and after thiopental and propofol induction, at intubation, and after intubation. B1, B2, and $\mathrm{B} 3$ refer to one, two, and three minutes before drug administration (control). I refers to $1.5 \mathrm{~min}$ after drug induction. Tl to T8 refer to one to eight minutes after intubation. An asterisk $(*)$ indicates $P<0.05$ when comparing the thiopental group with the propofol group.

cantly elevated in the thiopental group one minute after intubation when compared with the control $(P<$ 0.05 , Figure 2). There was no difference in the HR after intubation between groups.

\section{Discussion}

Our results suggest that at the doses studied, propofol provides a more stable anesthetic depth than thiopental after intubation in patients $>50 \mathrm{yr}$. Although both propofol and thiopental significantly decrease the AAI after anesthetic induction, tracheal intubation may stimulate patients and elevate the AAI in patients receiving thiopental, changing the AAI to a level indicating insufficient anesthetic depth. However, the AAI remained consistently low without rebound in the propofol group at a dose of $2 \mathrm{mg} \cdot \mathrm{kg}^{-1} \mathrm{iv}$. Similar reactions were shown for MBP and HR. After tracheal intubation, MBP and HR were relatively stable in the propofol group. However, in the thiopental group, tracheal intubation produced significant increases in MBP and HR.

Recently, the AAI was developed to assess the depth of anesthesia. ${ }^{6}$ Recent articles report that the AAI is an accurate indicator of the level of anesthetic depth and may be more valuable when monitoring depth of sedation than the bispectral index. ${ }^{7,8} \mathrm{We}$ 

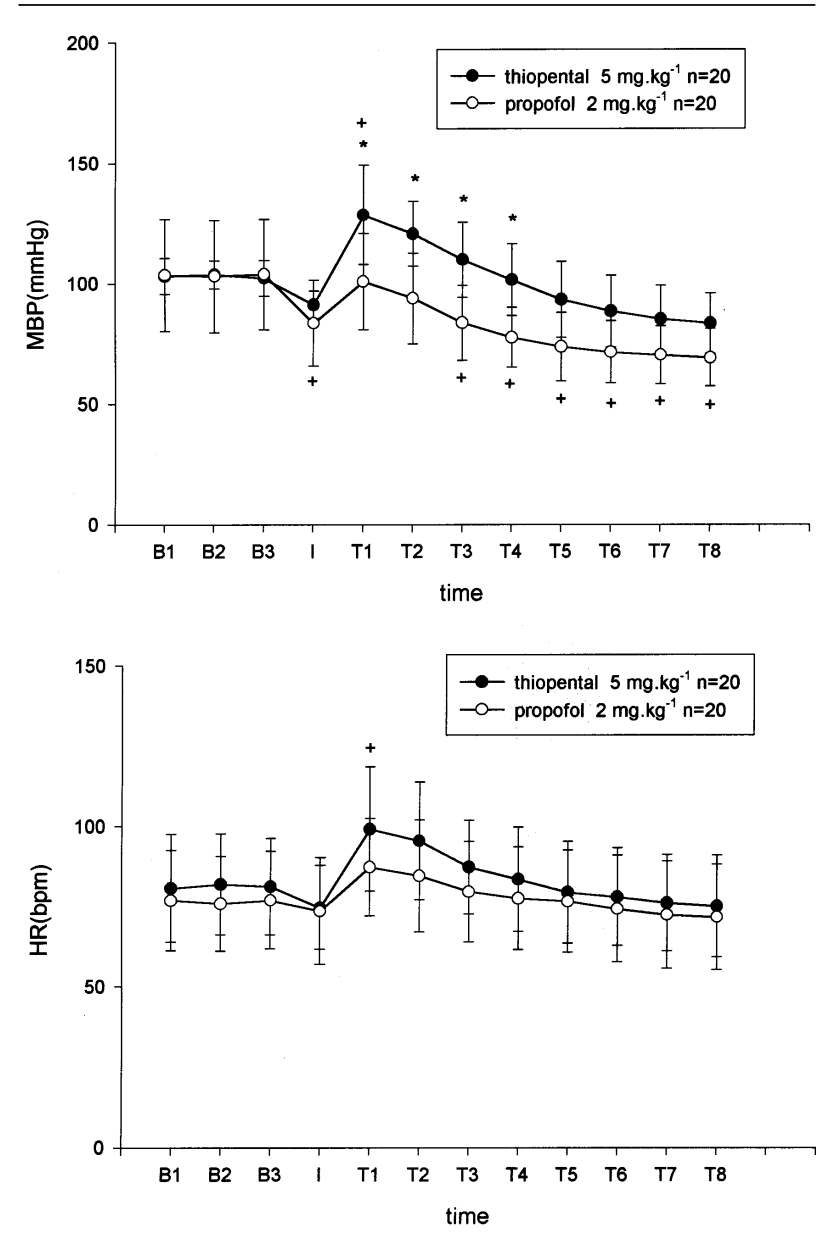

FIGURE 2 The mean blood pressure (MBP) (panel A) and heart rate (HR) (panel B) before and after thiopental and propofol induction, at intubation, and after intubation. B1, B2, and B3 refer to one, two, and three minutes before drug administration (control). I refers to $1.5 \mathrm{~min}$ after drug induction. Tl to T8 refer to one to eight minutes after intubation. An asterisk $\left({ }^{*}\right)$ indicates $P<0.05$ when comparing the thiopental group with the propofol group. A cross $(\dagger)$ indicates $P<0.05$ when compared with the control values.

chose this technique for continuous monitoring of anesthetic depth after induction of anesthesia and intubation.

Propofol and thiopental are both popular anesthetic induction agents. ${ }^{11,12} \mathrm{We}$ previously reported that tracheal intubation induced less hypertension and tachycardia when propofol was used for induction. Thiopental did not inhibit hypertension or tachycardia induced by tracheal intubation. ${ }^{9}$ Although cardiac inhibition and vasodilation from propofol may pro- duce hypotension and bradycardia, ${ }^{11-13}$ other commonly used general anesthetics, including thiopental, are similar. ${ }^{11,14,15}$ For example, Mouren et al. reported that propofol did not inhibit the myocardial performance of a blood-perfused isolated heart, but that thiopental did. ${ }^{16}$ Therefore, cardiac inhibition or vasodilation might not be the only factor in the inhibition of hypertension and tachycardia which can be found during tracheal intubation with propofol anesthesia. Inhibition of those actions by propofol may also originate in the central nervous system. Articles have reported that propofol is a good amnesic sedative and centrally inhibits the cardiovascular system especially in the cardiovascular control center, the medulla. ${ }^{17,18}$ Our results show that the AAI decreased rapidly after propofol or thiopental induction. However, the AAI increased in response to intubation in patients receiving thiopental. It appeared to be a reaction reflecting insufficient anesthetic depth, although patients did not completely regain consciousness. This reaction was not observed in the propofol group where the AAI remained low. Changes in blood pressure and heart rate paralleled changes in the AAI, suggesting that anesthetic depth is closely related to changes in BP and HR. However there is no evidence that the mechanisms by which noxious stimulation increase the AAI and $\mathrm{MBP} / \mathrm{HR}$ are the same.

In summary, we used the AAI to monitor anesthetic depth during induction of anesthesia and tracheal intubation, and demonstrated that propofol $2 \mathrm{mg} \cdot \mathrm{kg}^{-1}$ $i v$ provides a more stable anesthetic depth than does thiopental $5 \mathrm{mg} \cdot \mathrm{kg}^{-1} i v$ in patients $>50 \mathrm{yr}$. Propofol is also associated with less hypertension and tachycardia in response to tracheal intubation.

\section{References}

1 Mantzaridis H, Kenny GN. Auditory evoked potential index: a quantitative measure of changes in auditory evoked potentials during general anaesthesia. Anaesthesia 1997; 52: 1030-6.

2 Gajraj RJ, Doi M, Mantzaridis H, Kenny GN. Analysis of the EEG bispectrum, auditory evoked potentials and the EEG power spectrum during repeated transitions from consciousness to unconsciousness. Br J Anaesth 1998; 80: 46-52.

3 Gajraj RJ, Doi M, Mantzaridis H, Kenny GN. Comparison of bispectral EEG analysis and auditory evoked potentials for monitoring depth of anaesthesia during propofol anaesthesia. Br J Anaesth 1999; 82: 672-8.

4 Sebel PS, Heneghan CP, Ingram DA. Evoked responses-a neurophysiological indicator of depth of anaesthesia (Editorial). Br J Anaesth 1985; 57: 841-2. 
5 Iselin-Chaves IA, Moalem HE, Gan TJ, Ginsberg B, Glass PS. Changes in the auditory evoked potentials and the bispectral index following propofol or propofol and alfentanil. Anesthesiology 2000; 92: 1300-10.

6 Jensen EW, Nygaard M, Henneberg SW. On-line analysis of middle latency auditory evoked potentials (MLAEP) for monitoring depth of anaesthesia in laboratory rats. Med Eng Phys 1998; 20: 722-8.

7 Struys MM, Jensen EW, Smith W, et al. Performance of the ARX-derived auditory evoked potential index as an indicator of anesthetic depth. A comparison with bispectral index and hemodynamic measures during propofol administration. Anesthesiology 2002; 96: 803-16.

8 Ge SJ, Zhuang XL, Wang TT, Wang ZD, Li HT. Changes in the rapidly extracted auditory evoked potentials index and the bispectral index during sedation induced by propofol or midazolam under epidural block. Br J Anaesth 2002; 89: 260-4.

9 rang CY, Hsu JC, Lin CM, Huang SJ, Chung HS, Shyr $M H$. Hemodynamic responses of thiopental and propofol in different-aged patients during endotracheal intubation. Chang Gung Med J 2001; 24: 376-82.

10 Hines $W G$. Pragmatics of pooling in ANOVA tables. Am Statist 1996; 50: 127-39.

11 Vohra A, Thomas AN, Harper NJ, Pollard BJ. Noninvasive measurement of cardiac output during induction of anaesthesia and tracheal intubation: thiopentone and propofol compared. Br J Anaesth 1991; 67: 64-8.

12 Sellgren J, Ejnell H, Elam M, Ponten J, Wallin BG. Sympathetic muscle nerve activity, peripheral blood flows, and baroreceptor reflexes in humans during propofol anesthesia and surgery. Anesthesiology 1994; 80: 534-44.

13 Park WK, Lynch III C. Propofol and thiopental depression of myocardial contractility. A comparative study of mechanical and electrophysiologic effects in isolated guinea pig ventricular muscle. Anesth Analg 1992; 74: 395-405.

14 Lindgren L, Yli-Hankala A, Randell T, Kirvela M, Scheinin M, Neuvonen PJ. Hemodynamic and catecholamine responses to induction of anaesthesia and tracheal intubation: comparison between propofol and thiopentone. Br J Anaesth 1993; 70: 306-10.

15 GinT, O'Meara ME, Kan AF, Leung RK, Tan P, Yau $G$. Plasma catecholamines and neonatal condition after induction of anaesthesia with propofol or thiopentone at caesarean section. Br J Anaesth 1993; 70: 311-6.

16 Mouren S, Baron JF, Albo C, Szekely B, Arthaud M, Viars $P$. Effects of propofol and thiopental on coronary blood flow and myocardial performance in an isolated rabbit heart. Anesthesiology 1994; 80: 634-41.
17 Yang CY, Luk HN, Chen SY, Wu Chai CY. Propofol inhibits medullary pressor mechanisms in cats. Can J Anaesth 1997; 44: 775-81.

18 Krassioukov AV, Gelb AW, Weaver LC. Action of propofol on central sympathetic mechanisms controlling blood pressure. Can J Anaesth 1993; 40: 761-9. 